AperTO - Archivio Istituzionale Open Access dell'Università di Torino

Association between hospitalization-related outcomes, dynapenia and body mass index: The Glisten Study

This is the author's manuscript

Original Citation:

Availability:

This version is available http://hdl.handle.net/2318/1669582

since 2018-10-16T08:38:35Z

Published version:

DOI:10.1038/s41430-018-0184-0

Terms of use:

Open Access

Anyone can freely access the full text of works made available as "Open Access". Works made available under a Creative Commons license can be used according to the terms and conditions of said license. Use of all other works requires consent of the right holder (author or publisher) if not exempted from copyright protection by the applicable law.

(Article begins on next page) 


\section{Association between hospitalization-related outcomes, dynapenia and body mass index: The Glisten Study}

Rossi AP, Fantin F, Abete P, Bellelli G, Bo M, Cherubini A, Corica F, Di Bari M, Maggio M, Manca GM, Rizzo MR, Bianchi L, Landi F, Volpato S and the GLISTEN Group Investigators

\section{Abstract}

\section{Objective}

To compare the prognostic value of dynapenia, as evaluated by handgrip, and body mass index (BMI) on length of stay (LOS), days of bed rest, and other hospitalization-related outcomes in a population of older adults admitted to 12 italian acute care divisions.

\section{Methods}

Data on age, weight, BMI, comorbidities, ADL, physical activity level, muscle strength, were recorded at hospital admission. LOS, days of bed rest, intrahospital falls, and discharge destination were also recorded during the hospitalization. Subjects with BMI $<18.5 \mathrm{~kg} / \mathrm{m}^{2}$ were classified as underweight, subjects with BMI 18.5-24.9 as normal weight, subjects with BMI $\geq 25$ as overweight-obese.

\section{Results}

A total of 634 patients, mean age $80.8 \pm 6.7$ years and $49.4 \%$ women, were included in the analysis. Overall dynapenic subjects (D) showed a longer period of LOS and bed rest compared with non-dynapenic (ND). When the study population was divided according to BMI categories, underweight (UW), normal weight (NW), and overweight-obese (OW$\mathrm{OB}$ ), no significant differences were observed in hospital LOS and days of bed rest. When analysis of covariance was used to determine the difference of LOS across handgrip/BMI groups, D/OW-OB and D/UW subjects showed significantly longer LOS (11.32 and 10.96 days, both $p$ 0.05) compared to ND/NW subjects ( 7.69 days), even when controlling for age, gender, baseline ADL, cause of hospitalization and comorbidity. After controlling for the same confounding factors, D/OW-OB, D/NW and D/UW subjects showed significantly longer bed rest (4.7, 4.56, and 4.05 days, respectively, all $p 0.05$, but D/OW-OB $p 0.01$ ) compared to ND/NW subjects ( 1.59 days).

\section{Conclusion}

In our study population, LOS is longer in D/UW and D/OW-OB compared to ND/NW subjects and days of bed rest are mainly influenced by dynapenia, and not by BMI class. 


\section{Introduction}

Hospitalization related bed rest is considered as a primary factor determining functional decline in the elderly [1]. This decline frequently results in prolonged hospital stay, nursing home admission [2], and increased health care costs.

The concept of dynapenic obesity, defined as the combination of low muscle strength and obesity, has been proposed [3, 4], a condition associated with increased risk of worsening disability, hospitalization, falls and mortality [5,6].Taken separately, dynapenia and higher BMI previously showed an association with important hospitalization-related outcomes in older adults, such as length of stay (LOS) [7,8,9]. Obesity is associated with increased risk of longer hospital stay [7], which is one of the major determinants of hospital costs $[10,11]$.

Data on metabolic adaptations to bed rest have been collected and physical inactivity per se is one of the primary causes in the development of metabolic inflexibility mechanisms (insulin resistance, impaired lipid trafficking shift in muscle fiber type and ectopic fat storage) [8], but no studies have evaluated whether higher BMI is a risk factor for increased days of bed rest. Grip strength is a good marker of overall muscle performance and nutritional status and is a significant predictor of length of hospitalization, independent of age and gender [9].With the increasing rate of obesity and the potentially simultaneous development of sarcopenia, bed rest represents a difficult clinical challenge for elderly individuals.

Identification of patients at risk of prolonged hospital stay and bed rest might allow for the targeting of interventions and management of resources for the hospitalized elderly, but studies evaluating the combination of BMI and muscle strength to predict these outcomes are still lacking. The aim of this study was to compare the association between dynapenia, combined with low and high BMI and LOS, days of bed rest and other hospitalization-related outcomes in a population of older patients admitted to 12 acute care wards in Italy.

\section{Methods}

\section{Study design and data collection}

Data were collected from the Gruppo di Lavoro Italiano Sarcopenia-Trattamento e Nutrizione (GLISTEN) project, a multicenter italian observational study performed in geriatric and internal medicine acute care wards as reported elsewhere [12]. The aim of the study was to determine prevalence and clinical correlates of sarcopenia in older Italian hospitalized adults and to estimate incidence of sarcopenia during hospitalization, according to the EWGSOP definition, including muscle mass, gait speed and handgrip strength evaluation [13]. For this analysis only handgrip measurement was used. All patients consecutively admitted to participating geriatric units, between February and May 2014, were evaluated for enrollment. Exclusion criteria were: age $<65$ years and/or 
patient's reluctance to be involved in the study. All participants gave their written consent to participate to the study and were assessed within the first $48 \mathrm{~h}$ from hospital admission. All participating centers obtained ethical approval from their local ethical committee.

A standardized dedicated questionnaire including demographic characteristics, self-report functional status, cognitive, and mood assessment, medication use, incident and prevalent medical conditions and biochemical test results was used.

\section{Muscle strength assessment}

Handgrip muscle strength was assessed using a hand-held dynamometer (JAMAR hand dynamometer, Model BK-7498, Fred Sammons Inc., Brookfield, IL). The highest value of three trials for each hand was used in the analyses [14]. For the diagnosis of dynapenia, in accordance with Fried, BMI-adjusted cutoffs in men were $\leq 29 \mathrm{~kg}$ for subjects with $\mathrm{BMI} \leq 24 \mathrm{~kg} / \mathrm{m}^{2}, \leq 30 \mathrm{~kg}$ for subjects with BMI 24.1 to $28 \mathrm{~kg} / \mathrm{m}^{2}$ and $\leq 32 \mathrm{~kg}$ for subjects with BMI $>28 \mathrm{~kg} / \mathrm{m}^{2}$ [15]. In women, cutoffs were $\leq 17 \mathrm{~kg}$ for subjects with $\mathrm{BMI} \leq 23 \mathrm{~kg} / \mathrm{m}^{2}, \leq 17.3 \mathrm{~kg}$ for subjects with BMI 23.1 to $26 \mathrm{~kg} / \mathrm{m}^{2}, \leq 18 \mathrm{~kg}$ for subjects with BMI 26.1 to $29 \mathrm{~kg} / \mathrm{m}^{2}$ and $\leq 21 \mathrm{~kg}$ for subjects with BMI $>29 \mathrm{~kg} / \mathrm{m}^{2}$.

Of the initially screened 669 subjects, 14 were not included in the present analysis because grip strength was missing and 21 for missing height or weight data, leaving a final sample of 634 patients. Data on additional hospitalization-related outcomes (intrahospital falls and hospital discharge modality) were available for 615 subjects.

\section{Anthropometric assessment}

Subjects were weighed barefoot and wearing light indoor clothing to the nearest $0.1 \mathrm{~kg}$, and height was measured to the nearest $0.5 \mathrm{~cm}$ using a stadiometer. Body weight adjusted by stature $\left(\mathrm{kg} / \mathrm{h}^{2}\right)$ was used to give BMI. In case of bedridden patients, hospital patient lifts and flexible, inelastic measuring tape between the vertex of the head and the heel in the supine position were used. According to the NIH subjects with BMI $<18.5$ as underweight, subjects with BMI between 18.5 and 24.9 as normal weight, subjects with BMI 25 or greater as overweight-obese [16].

\section{Classification of groups}

Individuals were classified into six groups based on handgrip and BMI: non-dynapenic normal weight (ND/NW, considered as the reference group), dynapenic normal weight (D/NW), non-dynapenic underweight (ND/UW), dynapenic underweight (D/UW), nondynapenic overweight-obese (ND/OW-OB) and dynapenic overweight-obese (D/OW$\mathrm{OB})$.

\section{Covariates}

Sociodemographic variables (age, gender, smoking habits, education) were obtained from clinical interview at hospital admission. Functional status in basic activities of daily 
living (ADL), instrumental activity of daily living (IADL) and physical activity level (PASE) were assessed as reported elsewhere [12, 13].

Specific medical conditions diagnoses were recorded from patient or physicians interview and through a careful review of medical charts.

\section{Hospitalization related outcomes}

The principal outcome variable was hospital length of stay (LOS), i.e. the number of days between admission and discharge, with single day admissions coded as 0. Days of bed rest, intrahospital falls and modality of hospital discharge were also recorded.

\section{Statistical analysis}

For descriptive reasons, the characteristics of the study population were compared according to handgrip/BMI groups, using a chi-square test for categorical variables and the ANOVA or the non-parametric Wilcoxon Mann Whitney test for continuous variables with Bonferroni correction was applied to multiple comparison. ND/NW was the reference category.

Group differences in hospitalization related outcomes between dynapenic and nondynapenic subjects and across BMI classes were tested with one way-ANOVA and chisquare test for categorical variables. Covariance analysis was used to determine the difference of length of stay and days of bed rest across handgrip/BMI groups, after controlling for age, gender, baseline ADL, cause of hospitalization and active pathologies. In particular, the presence of fever, diarrhoea, hypertension, ischemic cardiomyopathy, atrial fibrillation, pulmonary embolism, thrombosis, orthostatic hypotension, peripheral vascular disease, cardiac failure, diabetes, hypothyroidism, hyperthyroidism, arthritis, osteoporosis, fractures, COPD, transient ischemic attack, stroke, hemiparesis, dementia, Parkinson disease, urinary infection, acute and chronic renal failure, prostatectomy, ovariectomy, metastasis, cancer, were considered as covariates in the model.

To investigate better the effect of the interaction of handgrip and BMI on LOS and bed rest days, linear regression analysis was performed including an interaction term to the model, which was calculated as the product of BMI and handgrip.

SPSS (version 21.0 for Window) was used to perform all analyses. 


\section{Results}

Characteristics of the 634 participants according to handgrip/BMI groups are presented in Table 1 . Mean age of study participants was $80.8 \pm 6.7$ years, and $49.4 \%$ were women.

Table 1 Selected general characteristics of study participants according to handgrip/BMI groups $(n=634)$

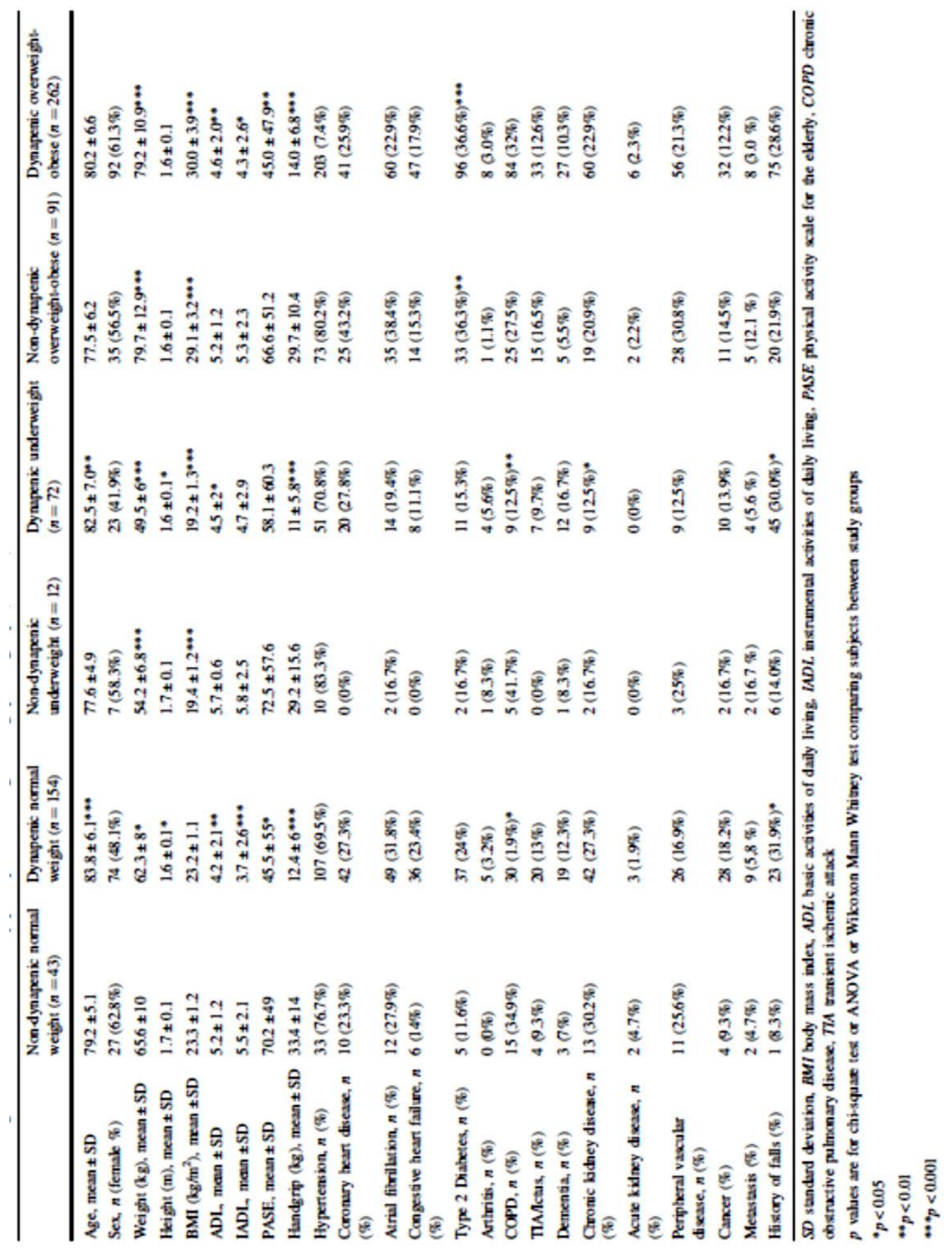


At hospital admission, D/NW subjects compared to ND/NW (the reference group) were older, showed higher prevalence of disability in ADL and IADL, lower PASE score, higher prevalence of COPD and history of falls.

D/OW-OB participants had higher prevalence of disability in ADL and IADL and lower PASE score compared to ND/NW. Prevalence of type 2 diabetes was significantly higher in $\mathrm{ND} / \mathrm{OW}-\mathrm{OB}$ and $\mathrm{D} / \mathrm{OW}-\mathrm{OB}$ subjects compared to the reference group.

D/UW participants had higher age, prevalence of ADL disability and history of falls, lower weight, height, BMI, handgrip and prevalence of COPD and chronic kidney disease.

Overall dynapenic subjects showed a longer period of hospital LOS and bed rest than the not-dynapenic counterpart (data not shown in Table).

Dividing the study population according to BMI (underweight, normal weight and overweight-obese) no significant differences were observed neither in hospital LOS nor days of bed rest (data not shown in Table). Table 2 shows the principal hospitalizationrelated outcomes according to handgrip/BMI groups. Using a Bonferroni correction, LOS was longer in $\mathrm{D} / \mathrm{UW}$ and $\mathrm{D} / \mathrm{OW}-\mathrm{OB}$, compared with ND/NW and days of bed rest were significantly higher in dynapenic subjects, independently of BMI class.

Table 2 Principal hospitalization related outcomes of study participants according to handgrip/BMI groups

\begin{tabular}{|c|c|c|c|c|c|c|}
\hline & $\begin{array}{l}\text { Nondyapenic normal } \\
\text { ueight }(n=41)\end{array}$ & $\begin{array}{l}\text { Dynpenic notral } \\
\text { weight }(n=149)\end{array}$ & $\begin{array}{l}\text { Noadynapenix } \\
\text { underweight }(n=12)\end{array}$ & $\begin{array}{l}\text { Dynaperic underweigth } \\
(n=72)\end{array}$ & $\begin{array}{l}\text { Non-dynapenic } \\
\text { overweiglx-obese }(n=89)\end{array}$ & $\begin{array}{l}\text { Dympenic ovenweiglx. } \\
\text { doese }(n=252)\end{array}$ \\
\hline $\begin{array}{l}\text { Days in hospital, mean } 1 \text { SD ( } n= \\
\text { 634) }\end{array}$ & $800 \pm 4.1$ & $11.1 \pm 93$ & $9.6 \pm 52$ & $109 \pm 5.7(9)$ & $9.69 \pm 7.7$ & $10.1 \pm 6.7^{*}$ \\
\hline Days in bed, $\operatorname{man} \pm \mathrm{SD}(n=634)$ & $15 \pm 2.8$ & $4.4 \pm 63^{*}$ & $26 \pm 37$ & $4.0 \pm 6.1\left({ }^{\bullet}\right)$ & $23 \pm 3.8$ & $4.6 \pm 6.2^{*}$ \\
\hline Ixrahospial falls $(\%)(n=615)$ & $0(0.0 \%)$ & $1(0.6 \%)$ & $1(83 \%)^{*}$ & $2(2.8 \%)$ & $0(0.05)$ & $13(5.1 \%)^{4}$ \\
\hline $\begin{array}{l}\text { Ixrinospital martality }(\%)(n= \\
615)\end{array}$ & $1(95 \%)$ & $2(0.1 \%)$ & $1(8.3 \%)$ & $1(1.4 \%)$ & $3(3.4 \%)$ & $5(2.0 \%)$ \\
\hline $\begin{array}{l}\text { Discharge no to usul residence } \\
\text { (\%) (death excluded) }(n=615)\end{array}$ & $4(95 \%)$ & $34(2.1 \%)^{4}$ & $1(8.36)$ & $18(250 \%)^{4}$ & $6(6.7 \%)$ & $19(7.5 \%)$ \\
\hline
\end{tabular}

$p$ values are for chisquas tes or Wikoson Mann Whiney tes conparing sabjats between stady goups

SD standard deviation

c $p<005$ 
A higher number of intrahospital falls in D/OW-OB and ND/UW was observed. Compared to the reference group, $\mathrm{D} / \mathrm{NW}$ and $\mathrm{D} / \mathrm{UW}$ showed a higher percentage of subjects that were not discharged at home after hospitalization. Finally, covariance analysis was used to compare differences in LOS across handgrip/BMI groups: D/UW and $\mathrm{D} / \mathrm{OW}-\mathrm{OB}$ still showed longer hospital stays compared to ND/NW subjects, even after controlling for age, gender, baseline ADL, cause of hospitalization and comorbidity (Fig. 1). D/OW-OB and D/UW subjects showed significantly longer LOS (11.32 and 10.96, both $p<0.05$ ) compared to ND/NW subjects (7.69 days). After controlling for the same confounding factors, dynapenic subjects, independent of BMI class, showed longer bed rest (Fig. 2). D/OW-OB, D/NW and D/UW subjects showed significantly longer bed rest $(4.7,4.56$, and 4.05 days, respectively, all $p<0.05$, but $\mathrm{D} / \mathrm{OW}-\mathrm{OB} p<0.01$ ) compared to ND/NW subjects (1.59 days). A not significant interaction between BMI categories and presence of dynapenia on LOS $(F=2.180, p=0.114)$ and days of bed rest $(F=1.337, p=0.263)$ was observed.

Fig. 1

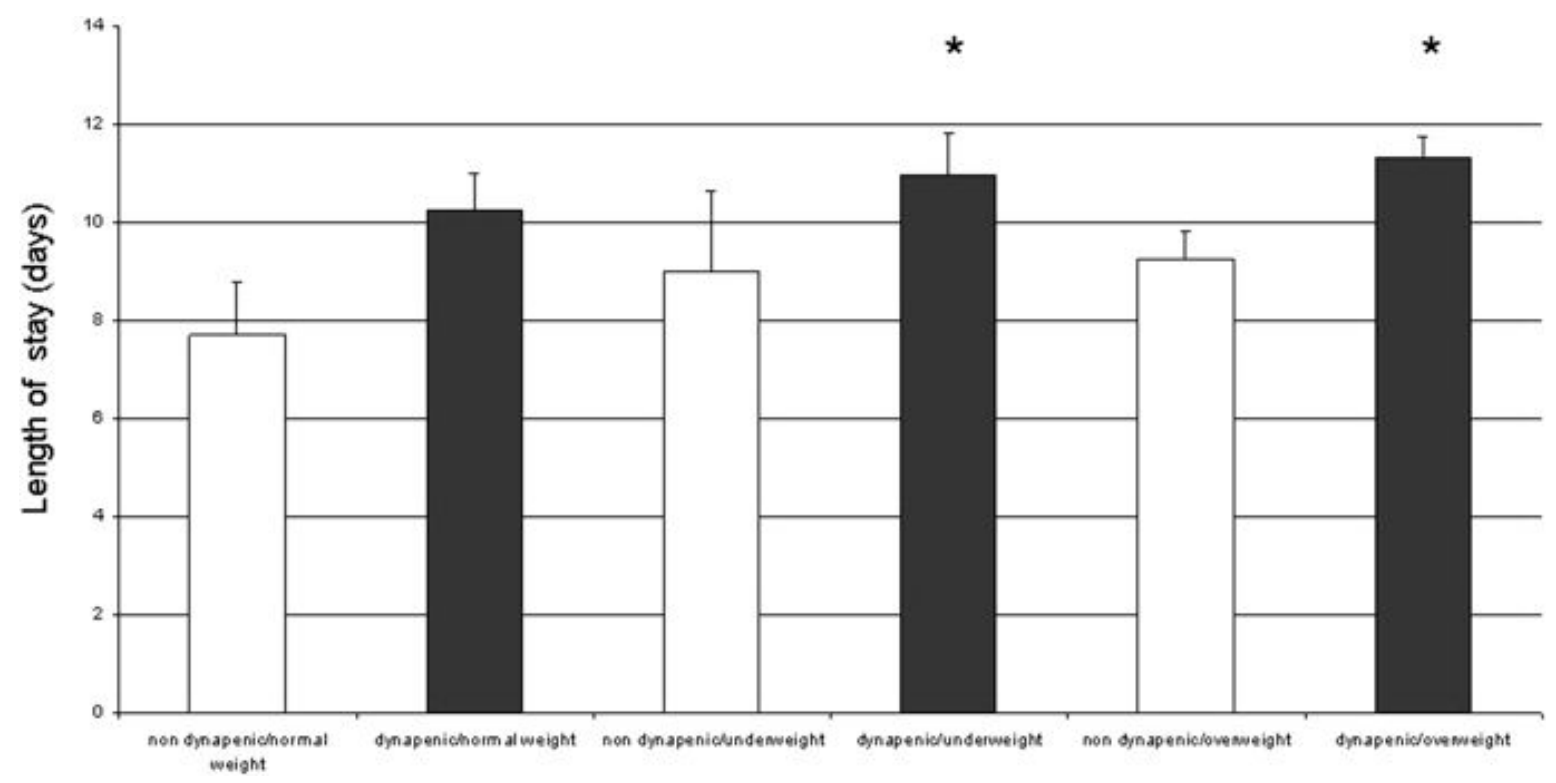

Length of stay across handgrip/BMI groups. Covariance analysis was used to compare differences in length of stay across handgrip/BMI groups, controlling for age, gender, ADL, cause of hospitalization and comorbidity. White bars non-dynapenic, Black bars dynapenic, reference category: non dynapenic/normalweight, * $p<0.05$ 
Fig. 2

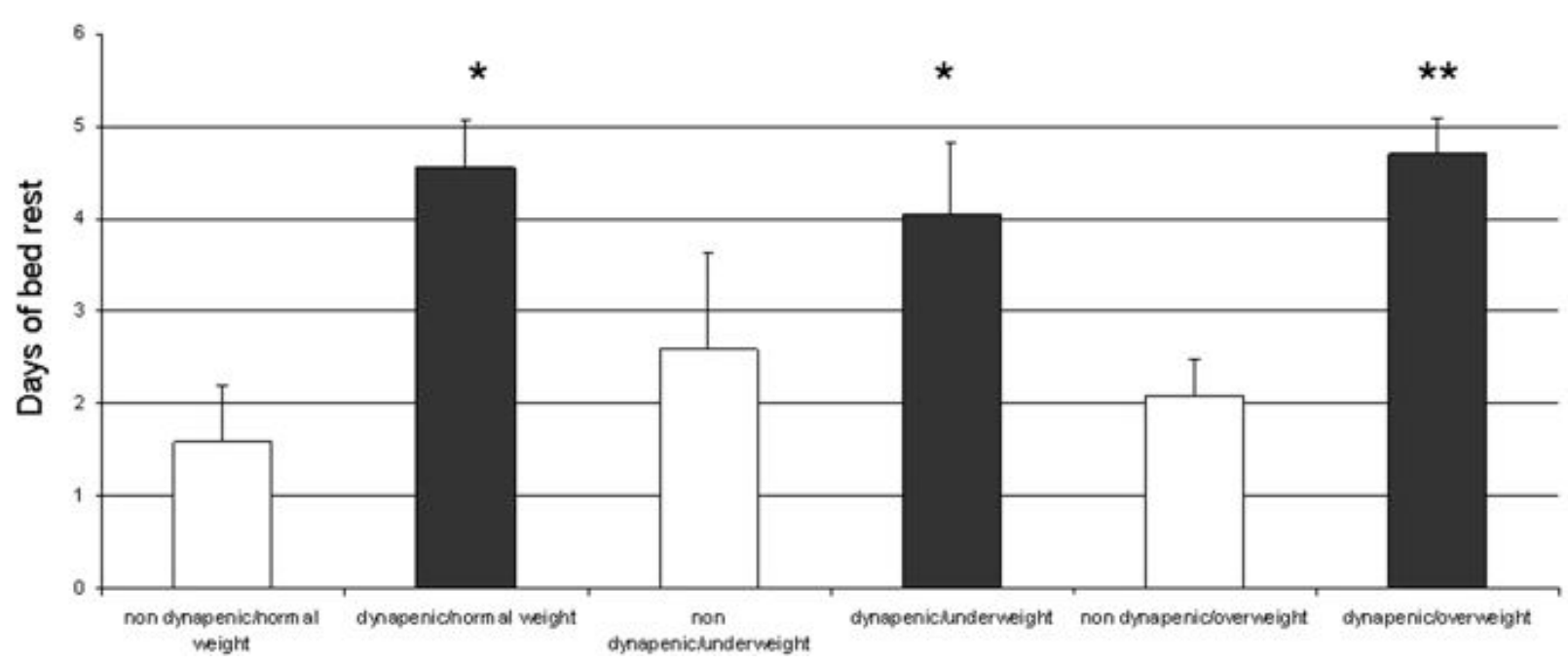

Days of bed rest across handgrip/BMI groups. Covariance analysis was used to compare differences in days of bed rest across handgrip/BMI groups, controlling for age, gender, ADL, cause of hospitalization and comorbidity. White bars non-dynapenic, Black bars dynapenic, reference category: non dynapenic/normalweight, * $\mathrm{p}<0.05 * * \mathrm{p}<0.01$

\section{Discussion}

Our study shows that LOS is longer in dynapenic patients with abnormal BMI (underweight and overweight-obese), compared to non-dynapenic normal weight subjects and that the days of bed rest during hospitalization are mainly influenced by dynapenia, and not by BMI class.

Dynapenic subjects had a longer hospital LOS and period of bed rest compared with nondynapenic subjects.

Previous studies have shown that grip strength measured in older people is a powerful predictor of functional decline, disability and mortality $[17,18,19]$, but only a few studies used this instrument in relation to LOS or other in-hospital prognostic purposes $[9,20,21]$.

Our results are partially in line with Matos et al. [20] that in medical and surgical patients showed an inverse association between grip strength and average LOS. Similar results were obtained by Roberts et al. [21] in older rehabilitation in-patients, admitted after acute medical illness, surgery or fracture.

After adjustment for confounding variables, dynapenic underweight, and overweightobese showed a longer hospital stay, compared with non-dynapenic normal weight subjects. Although BMI per se was not a risk factor for prolonged hospitalization, the 
reduced muscle strength associated with a BMI indicative of both, underweight or overweight, seems to identify a higher risk population.

Dynapenic subjects with high BMI are at higher risk of developing disability and rehospitalization [4], but no previous studies investigated whether this population also has a greater risk of unfavorable in-hospital outcomes. Our results are partially in line with Pichard et al. [22], who, in a population of nearly one thousand middle-aged subjects, found that the coexistence of low lean mass and high fat mass index was significantly associated with longer LOS $[22,23]$. Our study population, as compared to that of Pichard, was older, more homogeneous and our analysis was stratified on the basis of BMI and grip strength. In fact, we chose to consider handgrip, instead of quantitative muscle parameters, because it is well known that function decrease precedes muscle mass loss [24] and that in experimental models for disuse of muscle weakness, the relative contribution of muscular atrophy to weakness is modest [25]. Therefore, our population stratification has a dual advantage: first, use of easily available bedside measurements and, second, it is more sensitive in detecting the population at risk of unfavorable hospitalization-related outcomes.

Our results show that not only overweight, but also dynapenic underweight subjects are at risk of prolonged LOS and bed rest. Malnourished patients have slower healing, more complications, increased hospital costs and greater mortality [26]. Moreover, undernutrition is a powerful risk factor for loss of performance and sarcopenia development [27] and it tends to worsen during hospitalization [28], which partially explains the observed associations.

Dynapenic subjects showed a higher number of days of bed rest compared with nondynapenic normal weight subjects, independent of BMI classes. In previous reports, not only fat free mass, but also BMI was significantly associated with increased morbidity and LOS in hospitalized patients [22, 29], but a general lack of studies evaluating BMI as a risk factor for prolonged bed rest should be noted. In our study BMI was not associated with days of bed rest, but on the contrary, dynapenic subjects were bedridden for a significantly longer period of time. This is not surprising, considering that low muscle strength, is a well known risk factor for mobility limitations and poor physical performance in older adults [30,31].

Moreover, normal weight and underweight subjects with dynapenia showed a higher probability of not being discharged directly to usual residence. This observation confirms a previous report from Kerr et al. [9], which in a acute older medical in-patients study, showed a between grip strength adjusted for age and gender and the likelihood of discharge to usual residence, and also Armstrong et al. reported that in inpatient undergoing rehabilitation following stroke underweight subjects have a risk of the poorest hospital outcomes overall, with longer hospital stay and lower probability to be discharged back home [32, 33]. 
Some limitations of the present study should be acknowledged. First, the design of our study did not allow us to clarify any causal or temporal relationships between dynapenia, BMI and in-hospital outcomes. Second, BMI, in the elderly, has various deficiencies as a measure of obesity: its relation with body fat is not linear and may underestimate adiposity, as a consequence of age-dependent increase and redistribution of fat inside the body. However, BMI is a well-accepted surrogate of body fat and a simple measurement that, in combination with handgrip, allows us to identify a population at higher risk for unfavorable in-hospital outcomes. Last, it must be considered that cause of hospitalization includes also transient ischemic attack or stroke, conditions that may determine a possible reduction in handgrip strength test performance, although in our study the highest value of three trials for each hand was used in the analyses. Nevertheless, by stratifying the study population for ictus, non-significant differences in prevalence of dynapenia were observed in subjects with TIA/ictus.

In conclusion, in our study population, hospital stay is longer in dynapenic underweight, and overweight-obese subjects when compared to non-dynapenic normal weight and days of bed rest are mainly influenced by dynapenia, and not by BMI classes.

Some practical implications can be drawn from the results of the present study.

Dynapenic subjects are at higher risk of bed rest and longer hospital stay, therefore more attention in nutritional supplementation and mobilization during hospitalization must be given to this population. Moreover, in the dynapenic group, BMI seems to have a Ushaped association with hospital stay length, with both, underweight and overweight, significantly associated with longer hospitalization. Our study also confirms that nutritional status, in excess or defect, is associated with unfavorable hospital outcomes and support handgrip systematic evaluation in geriatric acute care settings.

\section{References}

1. Hoenig HM, Rubinstein LZ. Hospital associated deconditioning and dysfunction. J Am Geriatr Soc. 1991;39:220-2.

2. Fortinsky RH, Covinsky KE, Palmer RM, Landefeld CS. Effects of functional status changes before and during hospitalization on nursing home admission in older adults. J Gerontol Med Sci. 1999;54:M521-M526.

3. Stenholm S, Alley D, Bandinelli S, Griswold ME, Koskinen S, Rantanen T, et al. The effect of obesity combined with low muscle strength on decline in mobility in older persons: results from the InCHIANTI Study. Int J Obes. 2009;33:635-44. 
4. Rossi AP, Fantin F, Caliari C, Zoico E, Mazzali G, Zanardo M, et al. Dynapenic abdominal obesity as predictor of mortality and disability worsening in older adults: a 10-year prospective study. Clin Nutr. 2016;35:199-204.

5. Scott D, Sanders KM, Aitken D, Hayes A, Ebeling PR, Jones G. Sarcopenic Obesity and Dynapenic Obesity: 5-year associations with falls risk in middle-aged and older adults. Obesity. 2014;22:1568-74.

6. Rossi AP, Bianchi L, Volpato S, Bandinelli S, Guralnik J, Zamboni M, et al. Dynapenic abdominal obesity as a predictor of worsening disability, hospitalization, and mortality in older adults: results, from the InCHIANTI Study. J Gerontol A Biol Sci Med Sci. 2017;72:1098-104.

7. Akinyemiju T, Meng Q, Vin-Raviv N. Association between body mass index and in-hospital outcomes, Analysis of the nationwide inpatient database. Medicine. 2016;95:e4189.

8. Bergouignan A, Rudwill F, Simon C, Blanc S. Physical inactivity as the culprit of metabolic inflexibility: evidence from bed-rest studies. J Appl Physiol. 2011;111:1201-10.

9. Kerr A, Syddall H, Cooper C, Turner GF, Briggs RS, Sayer AA. Does admission grip strength predict length of stay in hospidlised older patient?. Age Aging. 2006;35:82-4.

10. Philbin E, McCullough P, Dec G, DiSalvo TG. Length of stay and procedure utilization are the major determinants of hospital charges for heart failure. Clin Cardiol. 2001;24:56-62.

11. Zhu H, Newcommon N, Cooper M, Green TL, Seal B, Klein G. Impact of a stroke unit on length of hospital stay and in-hospital case fatality. Stroke. 2009;40:18-23.

12. Bianchi L, Abete P, Bellelli G, Bo M, Cherubini A, Corica F, et al. Prevalence and clinical correlates of sarcopenia, identified according to the EWGSOP definition and diagnostic algorithm, in hospitalized older people: The Glisten Study. J Gerontol A Biol Sci Med Sci. 2017;72:1575-81.

13. Crutz-Jentoft AJ, Baeyens JP, Bauer JM, Boirie Y, Cederholm T, Landi F, et al. Sarcopenia: European consensus on definition and diagnosis. Report of the European Working Group on Sarcopenia in Older People. Age Aging. 2010;39:412-23.

14. Volpato S, Bianchi L, Cherubini A, Landi F, Maggio M, Savino E, et al. Prevalence and clinical correlates of sarcopenia in community-dwelling older people: application of the EWGSOP definition and diagnostic algorithm. J Gerontol A Biol Sci Med Sci. 2014;69:43846.

15. Fried LP, Tangen CM, Walston J, Newman AB, Hirsch C, Gottdiener J, et al. Frailty in older adults: evidence for a phenotype. J Gerontol A Biol Sci Med Sci. 2001;56:M1146-56.

16. World Health Organization. Physical Status: The Use and Interpretation of Anthropometry: Report of a WHO Expert Committee.. Geneva, Switzerland: World Health Organization; 1995. WHO Technical Report Series

17. Rantanen T, Guralnik JM, Foley D, Masaki K, Leveille S, Curb JD, et al. Midlife hand grip strength as a predictor of old age disability. JAMA. 1999;281:558-60.

18. Rantanen T, Harris T, Leveille SGK, Visser M, Foley D, Masaki K, et al. Muscle strength and body mass index as long-term predictors of mortality in initially healthy men. J Gerontol A Biol Sci Med Sci. 2000;55:M168-73.

19. Laukkanen P, Heikkinen E, Kauppinen M. Muscle strength and mobility as predictors of survival in 75-84-year-old people. Age Ageing. 1995;24:468-73. 
20. Matos LC, Tavares MM, Amaral TF. Handgrip strength as a hospital admission nutritional risk screening method. Eur J Clin Nutr. 2007;61:1128-35.

21. Roberts HC, Syddall HE, Cooper C, Aihie Sayer A. Is grip strength associated with length of stay in hospitalized older patients admitted for rehabilitation? Findings from the Southampton grip strength study. Age Ageing. 2012;41:641-6.

22. Pichard C, Kyle UG, Morabia A, Perrier A, Vermeulen B, Unger P. Nutritional assessment: lean body mass depletion at hospital admission is associated with increased length of stay. Am J Clin Nutr. 2004;79:613-8.

23. Kyle UG, Pirlich M, Lochs H, Schuetz T, Pichard C. increased length of stay in underweight and overweight patients at hospital admission: controlled population study. Clin Nutr. 2005;24:133-42.

24. Delmonico MJ, Harris TB, Visser M, Park SW, Conroy MB, Velasquez-Mieyer P, et al. Longitudinal study of muscle strength, quality and adipose tissue infiltration. Am J Clin Nutr. 2009;90:1579-85.

25. Clark BC, Fernhall B, Ploutz-Snyder LL. Adaptation in humans neuromuscular function following prolonged underweighting: I Skeletal muscle contractile properties and applied ischemia efficacy. J Appl Physiol. 2006;101:256-63.

26. Gomes F, Emery PW, Weekes CE. Risk of Malnutrition is an indipendent predictor of mortality, length of hospital stay, and hospitalization costs in stroke patientes. J Stroke Cerebrovasc Dis. 2016;25:799-806.

27. Rossi AP, Zanandrea V, Zoico E, Zanardo M, Caliari C, Confente S, et al. Inflammation and nutritional status as predictors of physical performance and strength loss during hospitalization. Eur J Clin Nutr. 2016;70:1439-42.

28. McWhirter JP, Pennington CR. Incidence and recognition of malnutrition in hospital. Br Med J. 1994;308:945-8.

29. Hauck K, Akinyemiju T, Meng O, Vin-Ravi N. Association between body mass index and inhospital outcomes Analysis of the nationwide inpatient database. Medicine. 2016;95:1-8.

30. Manini TM, Visser M, Won-Park S, Patel KV, Strotmeyer ES, Chen H, et al. Knee extension strength cutpoints for maintaining mobility. J Am Geriatr Soc. 2007;55:451-7.

31. Visser M, Goodpaster BH, Kritchevsky SB, Newman AB, Nevitt M, Rubin SM, et al. Muscle mass, muscle strength, and muscle fat infiltration as predictors of incident mobility limitations in well-functioning older persons. J Gerontol A Biol Sci Med Sci. 2005;60:32433.

32. Padwal RS, Wang K, Sharma AM, Dyer D. The impact of severe obesity on post acute rehabilitation efficiency, length of stay, and hospital costs. J Obes. 2012;2012:972365.

33. Armstrong R, Wang H, Dessureault L. Effect of body mass index on inpatient rehabilitation outcomes after stroke. 2013 Canadian stroke congress. Stroke. 2013;44:e174-e228. 